\title{
Optimal Dimensional Synthesis of a Symmetrical Five-Bar Planar Upper-Extremity Neuromotor Device
}

\author{
GAO Jianshe ${ }^{1}$, LI Mingxiang ${ }^{1}$, ALLISON Garry ${ }^{2}$, and CUI Lei ${ }^{3 *}$ \\ 1 School of Mechanical Engineering, Zhengzhou University, Zhengzhou 450001, China \\ 2 School of Physiotherapy and Exercise Science, Curtin University, Bentley 6102, Australia \\ 3 School of Civil and Mechanical Engineering, Curtin University, Bentley 6102, Australia
}

\begin{abstract}
Individuals with hemiplegia suffer from impaired arm movements that appear as a marked change in arm stiffness. A quantitative measure of arm stiffness would characterize rehabilitation therapy effectively, while little mechanism is designed to implement the function. A symmetrical five-bar linkage consisting of two revolute joints and three prismatic joints is presented. Inverse kinematics and forward kinematics are obtained first. Then inverse singularities and direct singularities of the mechanism are gained. Based on the results of kinematics analysis, the global stiffness index is defined. Finally, optimal dimensional synthesis of the mechanism in terms of maximum stiffness is conducted by genetic algorithms. The calculation results shows that when length of both the two linkage $a=830 \mathrm{~mm}$, interacting angle of the two guides $2 \delta=4.48$ radian, and maximum range of displacement of the two carriers $d_{\max }=940 \mathrm{~mm}$, the mechanism achieves highest rigidity and its workspace is singularity-free, which covers the human left and right arm range of motion. The proposed novel mechanism featuring high rigidity and a singularity-free workspace can provides rehabilitation training, but also solves the problem of quantitative measure of arm stiffness.
\end{abstract}

Keywords: Neuromotor, hemiplegia, rehabilitation, upper-extremity, parallel manipulator, five-bar linkage

\section{Introduction}

Stroke is a significant source of mortality and long-term disability in the developed world ${ }^{[1]}$. Many clinical trials have showed that intensive and specified tasks are the main factors in an effective treatment-program after stroke ${ }^{[2]}$. Individuals with hemiplegia often need consistent and extensive physical therapy, and these simple but repetitive exercises required full assistance from a physical therapists $^{[3-4]}$, hence the importance of robot-assisted rehabilitation training.

Robot-assisted rehabilitation training is fast becoming a common feature in rehabilitation. Since the pioneering study of the MIT-Manus ${ }^{[5]}$, the number of research groups developing robotic therapy devices has rapidly increased, and various devices have been developed for after-stroke automating training. LOPEZ $\mathrm{R}$, et $\mathrm{al}^{[6-7]}$, presented an exoskeleton robot focused on the rehabilitation of ankle and knee for the right leg. AKDOGAN and $\mathrm{ADLI}^{[8]}$ designed a therapeutic robot for lower limb rehabilitation. SHI, et $\mathrm{al}^{[9]}$, proposed an exoskeletal lower limbs rehabilitation robot and analyzed its dynamic characteristic. Among the devices, many devices are also developed for the rehabilitation of the upper extremity ${ }^{[10-13]}$. The initial results of robot-assisted rehabilitation are promising: patients who receive more therapy with a robotic device recover more movement ability ${ }^{[14-17]}$.

Individuals with hemiplegia suffer from impaired arm movements, appearing as a set of stereotypical kinematic and dynamic patterns due to abnormal joint coupling and muscles' resistance to passive elongation or stretch, leading to a marked change in tone, which clinicians traditionally judge subjectively.

An object measure of arm stiffness would yield more insight into the effect of stroke on a hemiplegic limb and better characterization of rehabilitation therapy. However the current measures of arm stiffness require patients performing repetitive test movement in several designated positions during functional tasks. This greatly limits their clinical use, since individuals with hemiplegia have lower fatigue threshold: many patients show increasing variety when repeating the same tasks ${ }^{[18-19]}$.

Five-bar linkages find many applications as a positioning device in a two dimensional planar space due to its high stiffness and lightweight ${ }^{[20-22]}$. The simplicity of five-bar symmetrical parallel mechanism has attracted the attention of many researchers, and many efforts have been contributed to the optimum design. For example, a solution space that solved the optimum design by means of performance atlases was developed ${ }^{[23-25]}$. A space made up of two normalized geometric parameters was established to show the characteristics of workspace and singularity ${ }^{[26-27]}$.

In many applications of parallel manipulators, the design 
workspace is required to cover a given workspace ${ }^{[28-29]}$ and the dimensions of the manipulators are optimized accordingly. RAO and $\mathrm{RAO}^{[30]}$ presented the dimensional synthesis of a 3-RPS (revolute-prismatic-spherical) parallel manipulator according to the limitation on the range of motion of the spherical joints: minimizing the total error of 10 positions in the sense of least-squares. MERLET ${ }^{[31]}$ proposed an algorithm to determine all the possible geometries of Gough-type 6-DOF parallel manipulators for a desired workspace, taking into account the leg-length limits, the mechanical limits on the passive joints, and interference between links. OTTVIANO and CECCARELLI $^{[32]}$ presented a formulation for an optimum design for CaPaMan architecture when the orientation workspace is suitably specified: the sum of the link lengths was minimized. KOSINSKA, et $\mathrm{al}^{[33]}$, designed the geometrical parameters of a Delta parallel manipulator for a specified workspace. AFFI, et $\mathrm{al}^{[34]}$, dealt with the synthesis and optimization of the workspace of a 3-translational-DOF parallel manipulator for a desired workspace. MERLET and DANEY ${ }^{[35]}$ considered a parallel robot that had to move within a given workspace and determined geometries of the robot, ensuring that these positioning errors lay within pre-specified limits for any pose of the robot in its workspace. LARIBI, et $\mathrm{al}^{[36]}$, presented an optimal dimensional synthesis method of the DELTA parallel robot for a prescribed workspace and genetic algorithms were used to find the dimensions of the DELTA robot.

This paper aimed to design a symmetric $2 \mathrm{P} 3 \mathrm{R}$ parallel manipulator as a neuromotor device that can measure the arm stiffness of an individual with hemiplegia and provide neuromotor rehabilitation training. Starting from the kinematics of the parallel manipulator, the paper adopts genetic algorithms to optimize the dimensions of the manipulator, whose workspace covers the target workspace of the left and right human arm. Further, no singularities reside within the workspace with maximum stiffness measurement.

The paper is organized as follows. Section 2 presents the forward and inverse kinematics and geometry of the neuromotor device. Section 3 discusses the singularities and global stiffness index. Section 4 derives the global stiffness index. Section 5 considers a human left and right arm workspace. Section 6 presents an optimal formulation to obtain the dimensions of the neuromotor device and the outcome of the optimization. Section 7 concludes the paper.

\section{Kinematics and Geometry of the Neuromotor device}

Fig. 1 shows a schematic of the pro-posed device being used by a human.

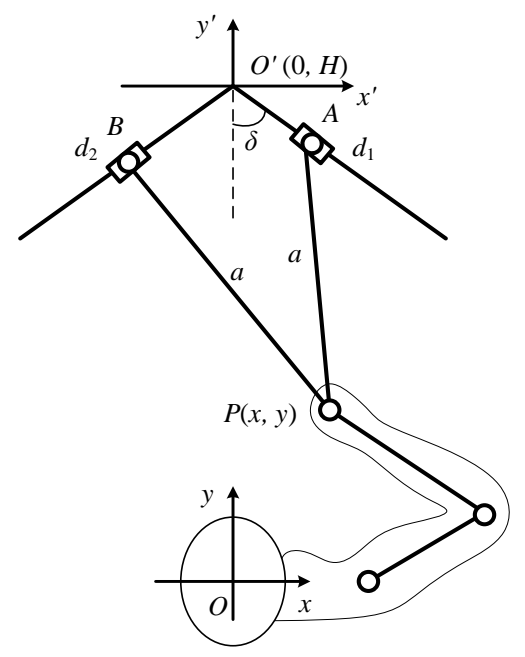

Fig. 1. Neuromotor device for upper-limb rehabilitation

The proposed neuromotor device is a symmetric five-bar PPRRR mechanism, where the interacting angle of the two guides is $2 \delta(0<\delta<\pi)$, the displacement of the carrier $A$ from the intersecting point $O^{\prime}$ is $d_{1}$, and the displacement of carrier $B$ from the intersecting point $O^{\prime}$ is $d_{2}$, the length of both the two linkage is $a$.

In the local frame $O^{\prime}-x^{\prime} y^{\prime}$, the coordinates of the carriers $A$ and $B$ are:

$$
\left\{\begin{array}{l}
x_{A}=d_{1} \sin \delta \\
y_{A}=-d_{1} \cos \delta
\end{array},\left\{\begin{array}{l}
x_{B}=-d_{2} \sin \delta \\
y_{B}=-d_{2} \cos \delta
\end{array} .\right.\right.
$$

The leg length gives two constraint equations as:

$$
\left\{\begin{array}{l}
\left(x-d_{1} \sin \delta\right)^{2}+\left(y+d_{1} \cos \delta\right)^{2}=a^{2} \\
\left(x+d_{1} \sin \delta\right)^{2}+\left(y+d_{1} \cos \delta\right)^{2}=a^{2}
\end{array} .\right.
$$

The inverse kinematics, i.e., solving for $d_{1}$ and $d_{2}$ for a given position $P$, can be obtained as:

$$
\begin{aligned}
& d_{1}=x \sin \delta-y \cos \delta \pm \sqrt{a^{2}-(x \sin \delta+y \sin \delta)^{2}} \\
& d_{2}=-x \sin \delta-y \cos \delta \mp \sqrt{a^{2}-(x \sin \delta-y \sin \delta)^{2}}
\end{aligned}
$$

Geometrically, the four solutions correspond to the following situations: any combination of $\left(A_{i}, B_{i}\right), i=1,2$, gives one of the four solutions.

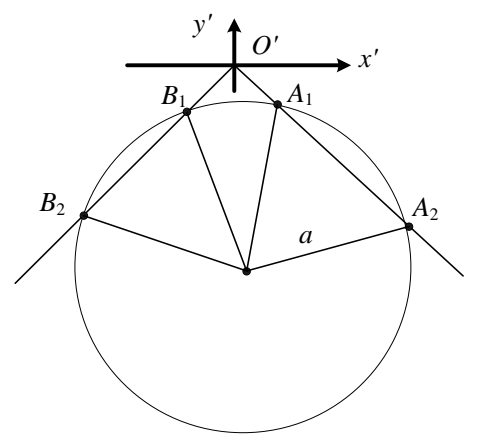

Fig. 2. Inverse displacement solution of the device 
The forward kinematics, i.e., solving for $x$ and $y$ for given $d_{1}$ and $d_{2}$, can be obtained as:

$x=\frac{d_{1}-d_{2}}{2}\left(\sin \delta \pm \cos \delta\left(\frac{4 a^{2}-d_{1}^{2}-d_{2}^{2}+2 d_{1} d_{2} \cos 2 \delta}{d_{1}^{2}+d_{2}^{2}-2 d_{1} d_{2} \cos 2 \delta}\right)^{1 / 2}\right)$,

$y=-\frac{d_{1}+d_{2}}{2}\left(\cos \delta \mp \sin \delta\left(\frac{4 a^{2}-d_{1}^{2}-d_{2}^{2}+2 d_{1} d_{2} \cos 2 \delta}{d_{1}^{2}+d_{2}^{2}-2 d_{1} d_{2} \cos 2 \delta}\right)^{1 / 2}\right)$

Geometrically, the two solutions $(+-,+)$ correspond to the following situations: the two intersecting points $P_{1}$ and $P_{2}$ of the circle centred at $A$ and the circle centred at $B$ with identical radius $a$ yield the two solutions. This corresponds to two configurations of the robot and only one is acceptable for a given configuration. From the setup of the configuration of the device, we shall always use the set $(+-)$, since $\delta$ is in the range of $(0, \pi)$ and the set with smaller $y$-coordinate corresponds to the setup.

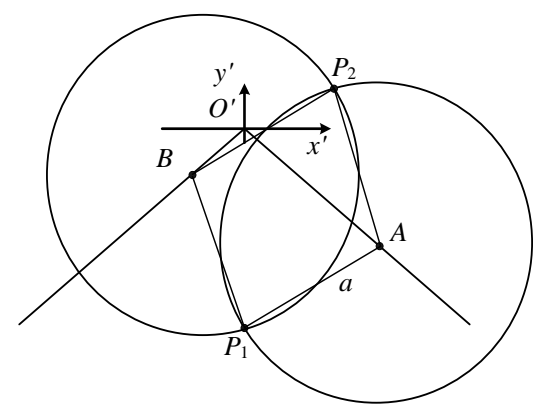

Fig. 3. Forward displacement solution of the device

\section{Jacobian Analysis: Inverse and Direct Singularities}

Differentiating Eq. (2) yields, the result is

$$
\begin{aligned}
& \left(x-d_{1} \sin \delta\right)\left(\dot{x}-\dot{d}_{1} \sin \delta\right)+\left(y+d_{1} \cos \delta\right)\left(\dot{y}+\dot{d}_{1} \cos \delta\right)=0 \\
& \left(x+d_{2} \sin \delta\right)\left(\dot{x}+\dot{d}_{2} \sin \delta\right)+\left(y+d_{2} \cos \delta\right)\left(\dot{y}+\dot{d}_{2} \cos \delta\right)=0
\end{aligned}
$$

Re-writing Eq. (5) in matrix form yields,

$$
\begin{aligned}
& \left(\begin{array}{cc}
d_{1}+y \cos \delta-x \sin \delta & 0 \\
0 & d_{2}+y \cos \delta+x \sin \delta
\end{array}\right)\left(\begin{array}{c}
\dot{d}_{1} \\
\dot{d}_{2}
\end{array}\right)= \\
& \left(\begin{array}{cc}
x-d_{1} \sin \delta & y+d_{1} \cos \delta \\
x+d_{2} \sin \delta & y+d_{2} \cos \delta
\end{array}\right)\left(\begin{array}{l}
\dot{x} \\
\dot{y}
\end{array}\right)
\end{aligned}
$$

Eq. (6) can be written as

$$
J_{q} \dot{q}=J_{x} \dot{x}
$$

where

$$
\begin{aligned}
& \boldsymbol{J}_{q}=\left(\begin{array}{cc}
d_{1}+y \cos \delta-x \sin \delta & 0 \\
0 & d_{2}+y \cos \delta+x \sin \delta
\end{array}\right) ; \\
& \dot{\boldsymbol{q}}=\left(\begin{array}{c}
\dot{d}_{1} \\
\dot{d}_{2}
\end{array}\right) ; \\
& \boldsymbol{J}_{x}=\left(\begin{array}{ll}
x-d_{1} \sin \delta & y+d_{1} \cos \delta \\
x+d_{2} \sin \delta & y+d_{2} \cos \delta
\end{array}\right) ; \\
& \dot{\boldsymbol{x}}=\left(\begin{array}{c}
\dot{x} \\
\dot{y}
\end{array}\right),
\end{aligned}
$$

where $\boldsymbol{J}_{q}$ is the inverse Jacobian, and $\boldsymbol{J}_{x}$ is the direct $\operatorname{Jacobian}^{[37]}$.

\subsection{Inverse Singularities}

The direct singularities occur when the determinant of the $\boldsymbol{J}_{q}$ is 0 :

$$
\left(d_{1}+y \cos \delta-x \sin \delta\right)\left(d_{2}+y \cos \delta+x \sin \delta\right)=0,
$$

That is,

$$
d_{1}+y \cos \delta-x \sin \delta=0 \text {, or } d_{2}+y \cos \delta+x \sin \delta=0
$$

From Eq. (3), we simplified the two functions above:

$$
\begin{aligned}
& a^{2}-(x \cos \delta+y \sin \delta)^{2}=0 \\
& a^{2}-(x \cos \delta-y \sin \delta)^{2}=0
\end{aligned}
$$

Then we obtained the equations of singularity line:

$$
\begin{aligned}
& x \cos \delta+y \sin \delta+a=0, \\
& x \cos \delta+y \sin \delta-a=0, \\
& x \cos \delta-y \sin \delta+a=0, \\
& x \cos \delta-y \sin \delta-a=0 .
\end{aligned}
$$

It could be seen that the singularities consist of four straight lines.

When the construction angle $\delta$ is between 0 to $\pi / 2$, the four singularities-lines can be shown in Fig. 4(a).

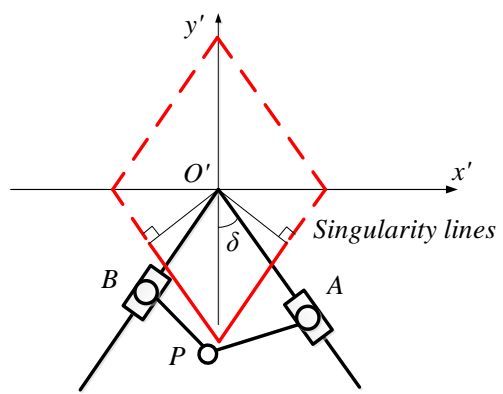

Fig. 4(a). Singularity lines when $\delta$ is between 0 to $\pi / 2$ 
When the construction angle $\delta$ is $\pi / 2$, the singularities-lines are degenerated into two parallel lines, as shown in Fig. 4(b).

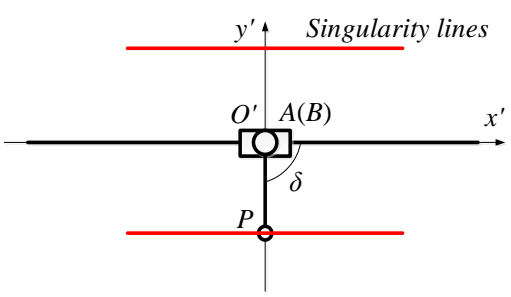

Fig. 4(b). Singularity lines when $\delta$ is equal to $\pi / 2$

When the construction angle $\delta$ is between $\pi / 2$ to $\pi$, the four singularities-lines can be shown in Fig. 4(c).

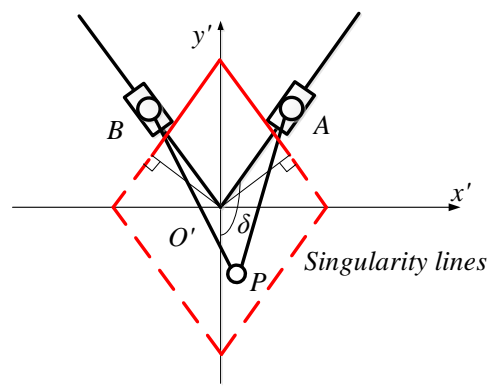

Fig. 4(c). Singularity lines when $\delta$ is between $\pi / 2$ to $\pi$

\subsection{Direct Singularities}

The direct singularities occur when the determinant of the $\boldsymbol{J}_{x}$ is 0 :

$$
\cos \delta\left(d_{1}-d_{2}\right) x+\sin \delta\left(d_{1}+d_{2}\right) y+d_{1} d_{2} \sin 2 \delta=0 .
$$

From Eq. (3) and Eq. (11), we obtained the equation of the singularity line:

$$
\frac{x^{2}}{a^{2} \tan ^{2} \delta}+\frac{y^{2}}{a^{2} \cot ^{2} \delta}=1 \text {. }
$$

The singularity consists of an ellipse, as in Fig. 5.

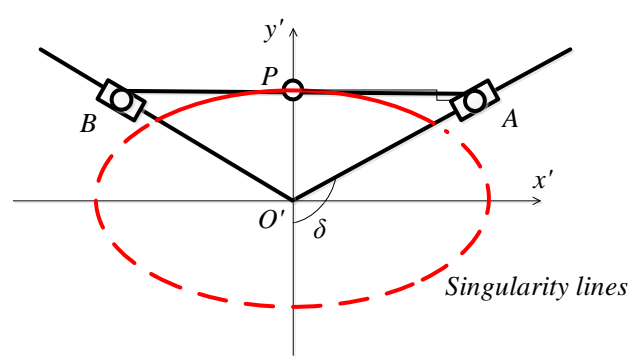

Fig. 5. Singularity lines is a part of a oval

\section{Global Stiffness Index}

To effectively measure the arm stiffness, the neuromotor device needs to have high stiffness so that the deformation on the effector is small when patients exert external force on it. The global stiffness index gives a quantitative measure.
First we defined an operating flexibility matrix $\boldsymbol{C}(\boldsymbol{q})$ that related the external force $\boldsymbol{F}$ to the output displacement $\Delta \boldsymbol{q}$ of the moving platform:

$$
\Delta q=C(q) F
$$

The square of the deformation modulus is

$$
\Delta \boldsymbol{q}^{\mathrm{T}} \boldsymbol{\Delta} \boldsymbol{q}=\boldsymbol{F}^{\mathrm{T}} \boldsymbol{C}(\boldsymbol{q})^{\mathrm{T}} \boldsymbol{C}(\boldsymbol{q}) \boldsymbol{F}
$$

Then we defined a parameter $\boldsymbol{K}$ as $\boldsymbol{K}=\boldsymbol{C}(\boldsymbol{q})^{\mathrm{T}} \boldsymbol{C}(\boldsymbol{q})$, where $\boldsymbol{C}(\boldsymbol{q})$ was the operating flexibility matrix. Re-writing Eq. (14):

$$
\Delta \boldsymbol{q}^{\mathrm{T}} \boldsymbol{\Delta} \boldsymbol{q}=\boldsymbol{F}^{\mathrm{T}} \boldsymbol{K} \boldsymbol{F}
$$

If we normalized the external torque to 1, that is, $\boldsymbol{F}^{\mathrm{T}} \boldsymbol{F}=1$, the maximum deformation could be obtained as

$$
\left\|q_{\max }\right\|=\sqrt{\max (\sigma)},
$$

where $\boldsymbol{\sigma}$ denotes the eigenvalues of the matrix $\boldsymbol{K}^{[32]}$.

The maximum global stiffness index can be defined as

$$
I=\int_{W}\left\|q_{\max }\right\| d W / \int_{W} d W
$$

This index represents the maximum deformation within the workspace.

\section{Human-Arm Workspace}

A human arm in this application can be modelled as $2 R$ series linkage and the contact between a human hand and the end-effector can be modelled as another $R$ joint, as in Fig. 1.

The average length of forearm with hand of males is $0.483 \mathrm{~m}$ and that of the upper arm $0.389 \mathrm{~m}$. The ranges of motions are $\theta_{R 1}$ from 0 to $3 / 4 \pi$ and $\theta_{R 2}$ from 0 to $3 / 4 \pi$. For the purpose of rehabilitation, it is reasonable to consider $\theta_{R 1}$ from $1 / 4 \pi$ to $3 / 4 \pi$. The kinematic parameters of the left arm can be obtained similarly: $\theta_{L 1}$ from $-3 / 4 \pi$ to $-1 / 4 \pi$ and $\theta_{L 2}$ from $-3 / 4 \pi$ to 0 . The average arm parameters are used in this paper $^{[38]}$.

If we account for both the left arm and the right arm, the desired workspace of the robot can be generated as: 


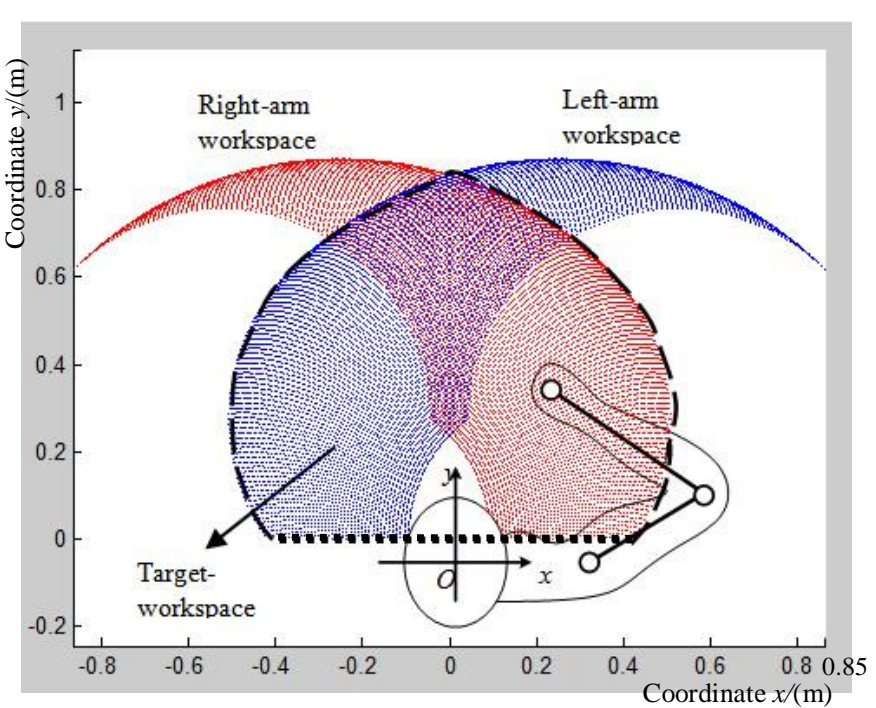

Fig. 6. Human-arm workspace

\section{Optimal Dimension Singularity-Free Workspace}

\subsection{Problem Formulation}

This section is to develop and solve the multidimensional, nonlinear optimization problem of selecting the geometric design variables for the neuromotor device to have the a specified workspace that is defined by a human's left and right arms. The proposed approach is based on the minimizing an objective function using nonlinear constrained optimization[34].

The optimal dimensional synthesis of the neuromotor device for the given workspace $W$ can be defined as follows:

Given: the human-arm workspace $W$;

Find: the parameter of the neuromotor device whose end-effector covers the given workspace $W$.

The parameters include the construction parameters $H$ and $\delta$, the actuation parameter $d_{\max }$, the leg length $a$. Please notice here that the physical construction determines the minimum actuation limit $d_{\text {min }}$ (we assumed a $100 \mathrm{~mm}$ offset from the point $O$ along the direction of the guide), as in Fig.7.

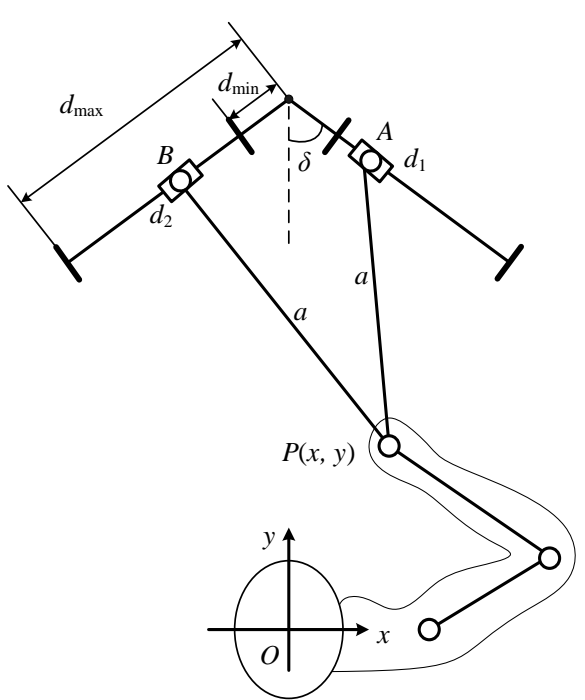

Fig. 7. Parameters of the neuromotor device

To make the device compact, we chose to minimize the maximum actuation range, i.e., the optimization problem with the parameters $\left(H, \delta, d_{\max }, a\right)$ for a suitably chosen objective function

$\min \quad \int_{W}\left\|q_{\max }\right\| d W / \int_{W} d W$

s.t. $\quad$ target workspace $\subset$ device workspace,

$$
\sigma_{i} \neq 0
$$

variables: $H, \delta, d_{\max }, a$.

We used the genetic algorithm in Global Optimization Toolbox from MATLAB to solve the problem, where the constraints were integrated via penalty functions. The initial population was set to contain 30 sets of each variables and the initial range was set as

$$
\begin{aligned}
& H \sim[200 \mathrm{~mm}, 1000 \mathrm{~mm}], \delta=[0, \pi], \\
& d_{\max } \sim[100 \mathrm{~mm}, 1000 \mathrm{~mm}], a \sim[500 \mathrm{~mm}, 1000 \mathrm{~mm}] .
\end{aligned}
$$

\subsection{Results}

All the results presented are obtained on an i5 processor of $3.30 \mathrm{GHz}$ with $8 \mathrm{~GB}$ memory. The calculation time is about 3.5 minutes. One set of optimal parameters is given as

$H=720 \mathrm{~mm}, a=830 \mathrm{~mm}, d_{\max }=940 \mathrm{~mm}, \delta=2.24 \mathrm{rad}$.

The workspace and the contour of $\left\|q_{\max }\right\|$ are shown in Fig.8 with contour lines representing the maximum deformation. 


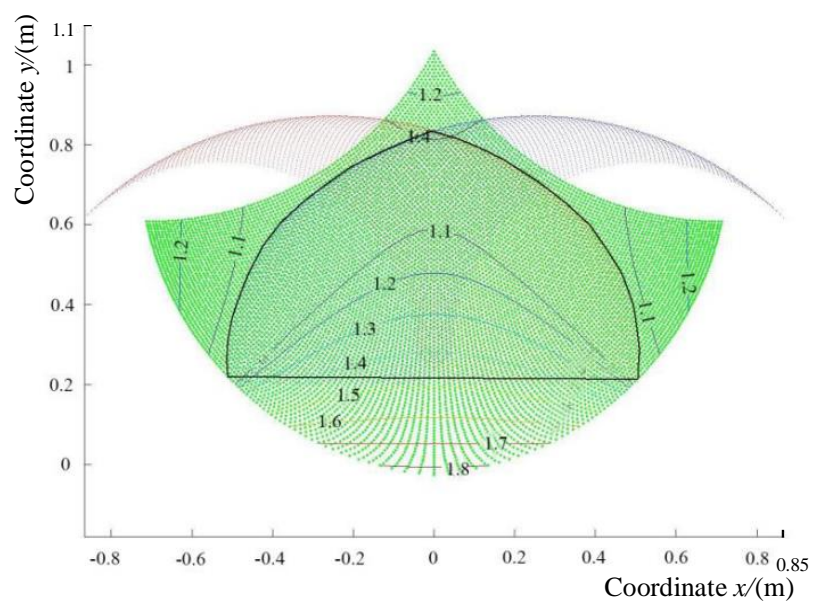

Fig. 8. Optimal workspace that covers the human-arm workspaces

The 3D model below approximates to $3 / 4 \pi$ to simplify the construction, without introducing any singularities.

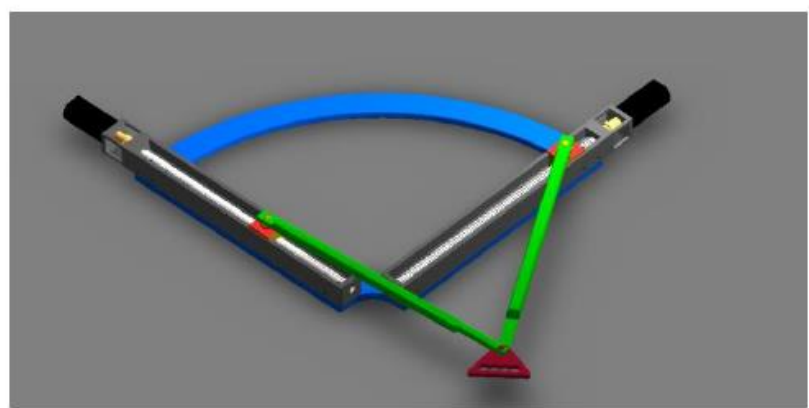

Fig. 8. 3D model of the optimal linkage

\section{Conclusions}

(1) A 2P3R planar upper-extremity neuromotor device with high stiffness for upper-limb rehabilitation is presented.

(2) The forward and inverse kinematics and singularities are obtained.

(3) Global stiffness index is defined; as a result, the arm stiffness of the device can be measured effectively.

(4) Genetic algorithms are adopted to achieve the optimal construction dimensions, leading to the workspace of the proposed device being singularity-free and covering a human left and right arm workspace.

\section{References}

[1] VASUDEVAN J M, BROWNE B J. Hemiplegic Shoulder Pain: An Approach to Diagnosis and Management[J]. Physical Medicine and Rehabilitation Clinics of North America, 2014, 25(2): 411-437.

[2] WINTER J M, CROME P, SIM J, et al. Effects of Mobilization and Tactile Stimulation on Chronic Upper-Limb Sensorimotor Dysfunction After Stroke[J]. Archives of Physical Medicine and Rehabilitation, 2013, 94(2): 693-702.

[3] WU Ming, LANDRY J M, KIM J, et al. Robotic Resistance/Assistance Training Improves Locomotor Function in Individuals Poststroke: A Randomized Controlled Study[J]. Archives of Physical Medicine and Rehabilitation, 2014, 95(5): 799-806.

[4] FARMER S E, DURAIRAJ V, SWAIN I, et al. Assistive Technologies: Can They Contribute to Rehabilitation of the Upper
Limb After Stroke?[J]. Archives of Physical Medicine and Rehabilitation, 2014, 95(5): 968-985.

[5] BUTEFISCH C, HUMMELSHEIM H, DENZLER P, et al. Repetitive training of isolated movements improves the outcome of motor rehabilitation of the centrally paretic hand[J]. Journal of the Neurological Sciences, 1995, 130(1): 59-68.

[6] LOPEZ R, AGUILAR H, SALAZAR S, et al. Adaptive Control for Passive Kinesiotherapy ELLTIO[J]. Journal of Bionic Engineering, 2014, 11(4): 581-588.

[7] LOPEZ R, AGUILAR H, SALAZAR S, et al. Modelado y Control de un Exoesqueleto para la Rehabilitación de Extremidad Inferior con dos grados de libertad[J]. Revista Iberoamericana de Automática e Informática Industrial RIAI, 2014, 11(3): 304-314.

[8] AKDOGN E, ADLI M A. The design and control of a therapeutic exercise robot for lower limb rehabilitation: Physiotherabot[J]. Mechatronics, 2011, 21(3): 509-522.

[9] SHI Xiaohua, WANG Hongbo, SUN Li, et al. Design and Dynamic Analysis of an Exoskeletal Lower Limbs Rehabilitation Robot[J]. Journal of Mechanical Engineering, 2014,50(3): 41-48. (in Chinese)

[10] HU Xiaoling, TONG Kaiyu, WEI Xijun, et al. The effects of post-stroke upper-limb training with an electromyography (EMG)-driven hand robot[J]. Journal of Electromyography and Kinesiology, 2013, 23(5): 1065-1074

[11] HU Xiaoling, TONG Kaiyu, LI Rui, et al. The effects of electromechanical wrist robot assistive system with neuromuscular electrical stimulation for stroke rehabilitation[J]. Journal of Electromyography and Kinesiology, 2012, 22(3): 431-439

[12] ZARIFFA J, KAPADIA N, KRAMER J L K, et al. Effect of a robotic rehabilitation device on upper limb function in a sub-acute cervical spinal cord injury population[C]// IEEE Int. Conf. on Rehabilitation Robotics (ICORR), Zurich, Switzerland, June 29-July 1, 2011: 1-5.

[13] PAN Lizheng, SONG Aiguo, XU Guozheng, et.al. Prescription Diagnosis for Upper-Limb Rehabilitation Training Robot Based on SVM-GDFNN[J]. Journal of Mechanical Engineering, 2014, 49(13): 17-22. (in Chinese)

[14] LO H S, XIE Shengquan. Exoskeleton robots for upper-limb rehabilitation: State of the art and future prospects[J]. Medical Engineering \& Physics, 2012, 34(3): 261-268.

[15] MAZZOLENI S, TURCHETTI G, PALLA I, et al. Acceptability of robotic technology in neuro-rehabilitation: Preliminary results on chronic stroke patients[J]. Computer Methods and Programs in Biomedicine, 2014, 116(2): 116-122

[16] HESSE S, SCHULTE-TIGGES G, KONRAD $M$, et al. Robot-assisted arm trainer for the passive and active practice of bilateral forearm and wrist movements in hemiparetic subjects[J]. Archives of physical medicine and rehabilitation, 2003, 84(6): 915-920.

[17] LO A C, GUARINO P D, RICHARDS L G, et al. Robot-Assisted Therapy for Long-Term Upper-Limb Impairment after Stroke[J]. New England Journal of Medicine, 2010, 362(19): 1772-1783.

[18] PALAZZOLO J J, FERRARO M, KREBS H I, et al. Stochastic Estimation of Arm Mechanical Impedance During Robotic Stroke Rehabilitation[J]. Neural Systems and Rehabilitation Engineering, IEEE Transactions on, 2007, 15(1): 94-103.

[19] PIOVESAN D, CASADIO M, MUSSA-IVALDI F A, et al. Multijoint arm stiffness during movements following stroke: Implications for robot therapy[C]// IEEE Int. Conf. on Rehabilitation Robotics (ICORR), Zurich, Switzerland, June 29-July 1, 2011: 1-7.

[20] CUI Lei, DAI Jiansheng. Reciprocity-Based Singular Value Decomposition for Inverse Kinematic Analysis of the Metamorphic Multifingered Hand[J]. ASME Journal of Mechanisms and Robotics, 2012, 4(3): 034502-034506.

[21] CUI Lei, DAI Jiansheng. Posture, Workspace, and Manipulability of the Metamorphic Multifingered Hand with an Articulated Palm[J]. ASME Journal of Mechanisms and Robotics, 2011, 3(2): 021001-021007. 
[22] DAI Jiangsheng, WANG Delun, CUI Lei. Orientation and Workspace Analysis of the Multifingered Metamorphic Hand-Metahand[J]. IEEE Transactions on Robotics, 25(4): 942-947.

[23] GAO Feng, LIU Xinjun, GRUVER W A. Performance evaluation of two-degree-of-freedom planar parallel robots[J]. Mechanism and Machine Theory, 1998, 33(6): 661-668.

[24] LIU Xinjun, WANG Qiming, WANG Jingsong. Kinematics, dynamics and dimensional synthesis of a novel 2-DoF translational manipulator[J]. Journal of Intelligent and Robotic Systems, 2005, 41(4): 205-224

[25] LIU Xinjun, WANG Jinsong, PRITSCHOW G. Performance atlases and optimum design of planar 5R symmetrical parallel mechanisms[J]. Mechanism and Machine Theory, 2006, 41(2): 119-144.

[26] CERVANTES-SANCHEZ J J, HERNANDEZ-RODRIGUEZ J C, RENDON-SANCHEZ J G. On the workspace, assembly configurations and singularity curves of the RRRRR-type planar manipulator[J]. Mechanism and Machine Theory, 2000, 35(8): 1117-1139.

[27] CERVANTES-SANCHEZ J J, HERNANDEZ-RODRIGUEZ J C, ANGELES J. On the kinematic design of the 5R planar, symmetric manipulator[J]. Mechanism and Machine Theory, 2001, 36(11-12): 1301-1313.

[28] CUI Lei, CUPCIC U, DAI Jiansheng. An Optimization Approach to Teleoperation of the Thumb of a Humanoid Robot Hand: Kinematic Mapping and Calibration[J]. ASME Journal of Mechanical Design, 2014, 136(9): 091005-091005.

[29] CUI Lei, WANG Delun, DAI Jiansheng. Kinematic Geometry of Circular Surfaces with a Fixed Radius Based on Euclidean Invariants[J]. ASME Journal of Mechanical Design, 2009, 131(10): 1010091-1010098.

[30] RAO N M, RAO K M. Dimensional synthesis of a spatial 3-RPS parallel manipulator for a prescribed range of motion of spherical joints[J]. Mechanism and Machine Theory, 2009, 44(2): 477-486.

[31] MERLET J P. Designing a Parallel Manipulator for a Specific Workspace[J]. The International Journal of Robotics Research, 1997, 16(4): 545-556.

[32] OTTAVIANO E, CECCARELli M. Optimal design of CaPaMan (Cassino Parallel Manipulator) with a specified orientation workspace[J]. Robotica, 2002, 20(2): 159-166.

[33] KOSINSKA A, GALICKI M, KEDZIOR K. Designing and optimization of parameters of delta-4 parallel manipulator for a given workspace[J]. Journal of Robotic Systems, 2003, 20(9): 539-548.

[34] AFFI Z, ROMDHANE L, MAALEJ A. Dimensional synthesis of a 3-translational-DOF in-parallel manipulator for a desired workspace[J]. European Journal of Mechanics - A/Solids, 2004, 23(2): 311-324

[35] MERLET J P, DANEY D. Dimensional Synthesis of Parallel Robots with a Guaranteed Given Accuracy over a Specific Workspace[C]// Proceedings of the 2005 IEEE International Conference on Robotics and Automation, Barcelona, Spain, 2005: 942-947.

[36] LARIBI M A, ROMDHANE L, ZEGHLOUL S. Analysis and dimensional synthesis of the DELTA robot for a prescribed workspace[J]. Mechanism and Machine Theory, 2007, 42(7): 859-870.

[37] TSAI W L. Robot Analysis: The Mechanics of Serial and Parallel Manipulators[M]. John Wiley \& Sons, 1999

[38] WINTER D A, Biomechanics and Motor Control of Human Movement $[\mathrm{M}]$. John Wiley \& Sons, 2009.

\section{Biographical notes}

GAO Jianshe, born in 1977, is currently an associate professor at school of mechanical engineering, Zhengzhou University, China. He received his doctor degree from Yanshan University, China, in 2006. His research interests include man-machine system and intelligent robotics.

Tel: +86-13837107816; E-mail: gao_jianshe@163.com

LI Mingxiang, born in 1991, a master's candidate of Mechanical and electrical integration at Zhengzhou University, China. He received his bachelor degree on mechatronics in Zhengzhou University, China, in 2009. His research interests include system control and intelligent robotics.

Tel: +86-13014589975; E-mail: li_mxiang@163.com

ALLISON Garry is currently a professor of Neuroscience and Trauma Physiotherapy with School of Physiotherapy and Exercise Science, Curtin University. He received his $\mathrm{PhD}$ from University of Western Australia. His research interests include neurosciences, rehabilitation and sport physiotherapy. Dr. Allison is on multiple Editorial boards and maintains his role as a researcher and academic mentor

Tel: +618 92662993 ; E-mail: G.Allison@ curtin.edu.au

CUI Lei is currently Lecturer in Mechatronics with Department of Mechanical Engineering, Curtin University. He received his $\mathrm{PhD}$ from University of London and then participated in the Autonomous Robotic Manipulation (ARM) Program sponsored by DARPA at Robotics Institute, Carnegie Mellon University. His research interests include robotic rehabilitation and biomimicry design for environmental monitoring.

Tel: +618 9266 7594; E-mail: Lei.Cui@ curtin.edu.au 